\title{
Segensbetrug oder Spaß? Thomas Manns Die Geschichten Jaakobs und die Genesis
}

Imposture ou plaisanterie? Les Histoires de Jaakob de Thomas Mann et la Genèse

Imposture or joke? Thomas Mann's The Tales of Jacob against the background of the Book of Genesis

\section{Charlotte Januel}

\section{(2) OpenEdition}

\section{Journals}

Édition électronique

URL : http://journals.openedition.org/ceg/1822

DOI : $10.4000 /$ ceg. 1822

ISSN : 2605-8359

\section{Éditeur}

Presses Universitaires de Provence

Édition imprimée

Date de publication : 1 décembre 2014

Pagination : 211-222

ISSN : 0751-4239

\section{Référence électronique}

Charlotte Januel, «Segensbetrug oder Spaß? Thomas Manns Die Geschichten Jaakobs und die Genesis », Cahiers d'Études Germaniques [Online], 67 | 2014, Online erschienen am: 17 Dezember 2017. abgerufen am 05 November 2020. URL : http://journals.openedition.org/ceg/1822 ; DOI : https:// doi.org/10.4000/ceg. 1822 


\title{
Segensbetrug oder Spaß? Thomas Manns Die Geschichten Jaakobs und die Genesis
}

\author{
Charlotte JANUEL
}

Aix-Marseille Université

Eine der bekanntesten Lug- und Truggeschichten der Weltliteratur dürfte wohl die Erschleichung des Erstgeburtsrechts durch Jakob im Alten Testament sein (1. Mose 27). Abraham, seinem Großvater, war von Gott versprochen worden, dass er Urvater eines großen Volkes werden solle, zahlreich „wie die Sterne am Himmel” (1. Mose 22, 17) und seinen Feinden überlegen. Isaak, sein Sohn, bekommt mit seiner Frau Rebekka Zwillinge: Esau und Jakob. Die Zwillinge könnten unterschiedlicher nicht sein: Esau, schon bei der Geburt ,rötlich und rauh wie ein Fell” wird zum Jäger, Jakob, „ein sanfter Mann”, bleibt „in den Hütten“ (1. Mose 25, 25-27). So ungleich wie die Eigenschaften ist auch die Elternliebe zwischen den Geschwistern verteilt: „Und Isaak hatte Esau lieb, und aß gern von seinem Weidwerk; Rebekka aber hatte Jakob lieb“ (1. Mose 25, 28).

Der Konflikt ist also vorprogrammiert: Durch Geburt ist Esau - wenn auch nur ganz geringfügig - der Ältere. Er wird außerdem von seinem Vater, auf dem der Segen Gottes liegt, vorgezogen. Er hat das Recht auf seiner Seite. Andererseits zeigen uns die Informationen, die wir zu seinem Lebenswandel bekommen, dass er dieses Erbe nicht so recht verdient, selbst wenn er formal der Ältere ist. Unsere Sympathie gilt also eher dem Jüngeren. Um das Ganze auf die Spitze zu treiben, erzählt uns die Bibel, dass Esau seine Erstgeburtsrecht um das sprichwörtlich gewordene Linsengericht an Jakob verkauft hat, wobei die völlig absurde Relation von Ware und Kaufpreis sofort ins Auge springt.

Als Isaak alt und inzwischen fast blind geworden ist, will er seinen Erstgeborenen segnen. Er ruft Esau zu sich, bittet ihn, ein Wild zu jagen und daraus ein Mahl zu kochen. Dies hört Rebekka. Sie ruft Jakob sofort zu sich und hat auch schon einen Plan: Jakob soll zwei Böcklein schlachten, und sie wird ihrem Mann dann ein Essen zubereiten, wie er es liebt. (1. Mose 27, 9). Damit soll Jakob zu seinem Vater gehen, sich für Esau ausgeben, und so den 
Segen erschleichen. Dieser hat zunächst Bedenken, die allerdings nicht grundsätzlicher Art sind: „Siehe, mein Bruder Esau ist rauh, und ich glatt; so möchte vielleicht mein Vater mich betasten und ich würde vor ihm geachtet, als ob ich ihn betrügen wollte; und brächte über mich einen Fluch und nicht einen Segen" (1. Mose 27, 12). Aber Rebekka hat auf alles eine Antwort; sie zieht ihrem Sohn Esaus beste Kleider an, so dass er wie sein Bruder riechen wird. Damit er sich auch anfühlt wie Esau, der Behaarte, legt Rebekka ihm vorsorglich das Fell des Böckleins um Hände und Hals. Mit möglichst allen ihm zur Verfügung stehenden Sinnen soll Isaak fühlen, riechen, schmecken, dass hier der Richtige seinen Segen erbittet. Einzig sein Gehör (sehen kann er ja nicht mehr) kann ihn noch zweifeln lassen - kann sich Jakob auch die Stimme Esaus zu eigen machen?

Der Plan funktioniert, obwohl Isaak Zweifel an der Identität seines Sohns anmeldet: „Die Stimme ist Jakobs Stimme, aber die Hände sind Esaus Hände“ (1. Mose 27, 22). Zweimal muss Jakob versichern, dass er wirklich Esau ist, bevor sein Vater ihn segnet. Als kurze Zeit später Esau mit dem von ihm gekochten Mahl beim Vater eintrifft, kann Isaak nur konstatieren: „Dein Bruder ist gekommen mit List, und hat deinen Segen hinweg“" (1. Mose 27, 35).

So wie diese Geschichte erzählt wird, kann sie als Schulbeispiel der Lüge verstanden werden. Peter von Matt widmet ihr ein Kapitel in seinem Buch Die Intrige - Theorie und Praxis der Hinterlist: „Diese Geschichte ist in höchstem Maße skandalös. Sie ist ein Bericht über Lug und Trug, wobei die göttliche Macht diesen Lug und Trug offensichtlich anerkennt.“1

Als Thomas Mann in den Zwanziger Jahren des vergangenen Jahrhunderts anfing, seinen großen Josephroman zu schreiben, konnte er davon ausgehen, dass die biblische Geschichte jedem seiner deutschen Leser bekannt war. Wie sie im Roman erzählt wird, finden wir in den Geschichten Jaakobs im vierten Hauptstück unter dem Titel „Der große Jokus“, das mit den Worten „in Wahrheit wurde niemand betrogen, auch Esau nicht" beginnt. ${ }^{2}$

„Jokus"? „Niemand wurde betrogen“? Wie kann Thomas Mann das behaupten, ohne seine zeitgenössischen Leser zu schockieren? Werden sie nicht diesen offensichtlichen Widerspruch zur Bibel als Verfälschung eines allseits bekannten Textes wahrnehmen? Wie wird es möglich, den Leser glauben zu machen, dass es sich hier um einen großen Spaß handelt, bei dem „die Leute johlen vor Lachen"? (S. 157). Welche Kniffe und Listen wendet der Autor an, um seinen Leser dahin zu lenken, wohin er ihn haben will?

Im Folgenden soll gezeigt werden, wie es in den Geschichten Jaakobs gelingt, dem Leser diese Umschreibung der biblischen Geschichte nicht nur glaubhaft zu machen, sondern ihn dahin zu führen, sie als eigentliche Version

\footnotetext{
${ }^{1}$ Peter von MATt, Die Intrige - Theorie und Praxis der Hinterlist, München, dtv, 2008, S. 110 .

2 Thomas MANN, Joseph und seine Brüder, Ausgabe in einem Band, Frankfurt/Main, S. Fischer Verlag, 1964, S. 148. Im folgenden wird auf diesen Text nicht durch Fußnoten, sondern durch Seitenangaben im Text verwiesen.
} 
anzunehmen, soll gezeigt werden, wie aus einer Lüge ein ungeheurer Spaß, aus den Folgen einer Betrugsgeschichte ein großer Jokus werden kann.

In dieser Perspektive werden das Verhältnis von Mythos und Individualität, die Charakterisierung der Hauptpersonen, die Rolle des Erzählers und die Erzählstruktur des Romans untersucht.

\section{Mondgrammatik}

Der Mond ist das dominierende Gestirn in diesem Roman. Sein weiches, diffuses Licht erzeugt eine andere Klarheit als die der Sonne. Er ist auch das Symbol der Wanderung; er begleitet Abraam, als dieser aus Ur auswandert, in seinem Licht sehen wir Joseph zum ersten Mal, und auch der Erzähler fühlt sich dem Mond verbunden: „Des Erzählers Gestirn - ist es nicht der Mond, der Herr des Weges, der Wanderer, der in seinen Stationen zieht, aus jeder sich wieder lösend?" (S. 39) Der Mond ist aber auch Mittler zwischen Oben und Unten, zwischen Sonne und Erde. „Sonne und Erde sind, jeweils für sich genommen, feindliches Gebiet ohne des Mondes Vermittlung. Zum Sonnenbereich gehört die Wüste, zum Erdbereich der Acker, zum Mond aber der Hirte und seine ziehende Herde. “ $^{3}$

Von Anfang an wird klargestellt, dass die Identität der Roman-Figuren nicht fest umrissen ist, dass die individuellen Züge von Jaakob und Esau sich vermischen mit Überlieferungen aus älteren Zeiten, in denen ähnliche Personen gleichen oder anderen Namens existierten und handelten und so den Nachfahren ihre Existenz und Handlungen gleichsam vorgaben. Dieses Verfahren wird in einem eigenen Kapitel im zweiten Hauptstück erklärt, das „Mondgrammatik“ überschrieben ist (S. 89-90). Die Klarheit der Sonne, in der alles eindeutig erscheint, wird hier der Klarheit des Mondes gegenübergestellt, „,ie damals und dort dem Geist als wahre Klarheit erschien“ (S. 89). Die Identität, die Individualität der handelnden Personen vermischt sich nämlich mit der ihrer Vorgänger, ihrer Ahnen, ihrer Urväter, und wenn sie von sich selbst sprechen, kann es durchaus sein, dass dieses Ich nicht so genau definiert war, wie es die grammatische erste Person glauben macht,

sondern gleichsam nach hinten offenstand, ins Frühere, außer seiner eigenen Individualität Gelegene überfloß und sich Erlebnisstoff einverleibte, dessen Erinnerungs- und Wiedererzeugungsform eigentlich und bei Sonnenlicht betrachtet die dritte Person statt der ersten hätte sein müssen (S. 90).

Im Roman wird diese „Mondgrammatik“ am Beispiel Eliezers erklärt. Dieser Eliezer ist Hausvogt und erster Knecht Jaakobs, aber seine Identität vermischt sich mit der eines anderen, viel älteren in der Bibel erwähnten

\footnotetext{
${ }^{3}$ Hermann KURZKE, Mondwanderungen - Wegweiser durch Thomas Manns JosephRoman, Frankfurt/Main, Fischer-Verlag, 2008, S. 19.
} 
Eliezer, Knecht Abraams. Auch Jaakobs Vater Jizchak ist nicht identisch mit dem Sohn Abrahams, selbst wenn er dessen Geschichte als die seinige erzählt, sei es, dass es sich um eine Imitation seines Urvaters handelt, oder weil er sie als seinem eigenen Leben zugehörig betrachtete.

Die Hauptpersonen in der Genesis erreichen alle ein „biblisches Alter“, so war zum Beispiel Abraham bei seinem Tod ,hundert und fünf und siebzig Jahre" (1. Mose 25, 7). Natürlich geht die Bibelforschung zur Entstehungszeit des Romans nicht mehr von solchen Altersangaben aus. Zwischen der Urvätergeschichte und der Ansiedlung eines Stammes Israel in Ägypten müssen ungefähr 600 Jahre gelegen haben. Die „Mondgrammatik“ macht diesen Zeitsprung deutlich, lässt ihn aber gleichzeitig wieder verschwimmen.

Jedenfalls unterscheiden die handelnden Personen „zwischen Ich und Nicht-Ich weniger scharf [...] als wir es zu tun gewohnt sind [...]" (S. 94). Diese Imitation, Wiederholung, Nachfolge nennt Thomas Mann „Mythus“. „Das Mythus vergegenwärtigt ein Urgeschehen. Einst hat irgendein Kain wirklich seinen Bruder Abel erschlagen. Die mythische Erzählung [...] bietet in der Folgezeit das Paradigma, das für alle ähnlich gelagerten Fälle als Modell dient." ${ }^{4}$

Gleichzeitig bietet die „Mondgrammatik“ aber auch die Möglichkeit, durch die Überlagerung verschiedener Identitäten den handelnden Personen Eigenschaften und Verhalten zuzuschreiben, die in der biblischen Vorlage so nicht gegeben sind und ihr vielleicht sogar widersprechen.

\section{Esau, Jaakob und Isaak}

Besonders deutlich und eindeutig negativ wirkt sich dieses Verfahren für Esau aus. Im biblischen Text wird Esau beschrieben als ,rötlich, rauh wie ein Fell“, „Edom der Rote“, ein Jäger und Liebling seines Vaters. Zum Leidwesen seiner Eltern hat er sich sehr jung mit kanaanitischen, d.h. ungläubigen, anderen Göttern anhängenden Frauen vermählt. Gegen eine warme Mahlzeit verschleudert er sein Erstgeburtsrecht und damit den Segen Gottes, da er mit dem auf Transzendenz ausgerichteten Glauben seiner Ahnen nichts anfangen kann: „Siehe, ich muss doch sterben; was soll mir denn die Erstgeburt"? (1. Mose 25, 32) Insgesamt erscheint er uns als etwas tumber, ungeschlachter, aber nicht bösartiger Mann.

Diese Vorgaben werden im Roman erklärt, erweitert, differenziert, aber auch durch entsprechende Charakterisierungen so zugespitzt, dass eigentlich kein gutes Haar an Esau bleibt und er von Anfang an als die lächerliche Person dargestellt wird, als die er sich dann im entscheidenden Kapitel „Der große Jokus" erweist. Als Jaakob ihn 25 Jahre nach dem Segensbetrug wiedersieht - die Brüder sind inzwischen 55 Jahre alt -, hat er sich weder physisch noch psychisch stark verändert. Seine Person scheint weder lern-

${ }^{4}$ Ibid., S. 24. 
noch entwicklungsfähig. Er spielt auf einer Rohrpfeife und tanzt dazu, und er wirkt dabei so lächerlich, dass man Jaakobs Gefühle von „Geringschätzung, Scham, Erbarmen und Abneigung" (S. 108) gegen seinen Bruder nur teilen kann. Jaakob war ,gründlich angewidert von Esau's Worten, fand sie höchst peinlich, hirnlos und liederlich" (S. 110) und überlegt nur, wie er diesen Bruder so schnell wie möglich wieder los wird, während Esau ihn herzt und küsst, so dass auch der Leser die Liebesbezeigungen des Älteren als unangenehm und ekelerregend empfindet.

Esau steht den Edomitern nahe, den „Leuten von Edom, zu denen Esau von früh an Beziehungen unterhalten hatte und zu denen er später vollends überging“ (S. 98). Später wird von ihm gesagt, dass er „Edom, der Rote“ ist (S. 140). Mit dieser Gegenwartsform wird die ,zeitlose und über-individuelle Zusammenfassung des Typus“. erklärt. Diese Beziehungen führen - für den Erzähler logisch - dazu, dass er nicht gesegnet, sondern verflucht wird: „Das beweist, dass es sich bei Segen oder Fluch nur um Bestätigungen handelt, dass sein Charakter, das heißt seine Rolle auf Erden, von langer Hand her festgelegt“ war (S. 99). Diese Rolle ,als sonnverbrannter Sohn der Unterwelt" muss er spielen, ,aus mythischer Bildung und Gehorsam gegen das Schema“" (S. 99).

Natürlich ist er auch in Glaubensdingen ein Paria. Schon dass er seine Frauen unter den Kanaaniterinnen suchte - „Baalsanbeterinnen“ (S. 98) schreibt der Erzähler -, empört und betrübt seine Eltern. Mit fortschreitendem Alter entfernt er sich immer weiter vom Glauben an Abraams Gott. Bei seiner Begegnung mit Jaakob wird er durch sein Flötenspiel in die Nähe von Pan gerückt.

Auch sein Verhältnis zu Ismael, seinem Onkel, wird äußerst kritisch gesehen. In der Bibel wird es nur mit einem Vers erwähnt (er ging „,zu Ismael, und nahm [...] Mahalath, die Tochter Ismaels, des Sohnes Abrahams [...] zum Weibe“, 1. Mose 28, 9). Im Roman werden daraus Mordpläne, die Ismael seinem Neffen und Schwiegersohn vorschlägt: Esau solle seinen Vater Isaak ermorden, dann werde er, Ismael „,dem Jaakob einen Pfeil so genau in den Nacken schießen, dass er durch den Kehlkopf wieder herauskomme“ (S. 159). Dieses Komplott gelangt allerdings nicht zur Ausführung, weil Esau dann doch Bedenken kommen.

Aber auf diese Weise gelingt es dem Erzähler, Esau nicht nur als in Glaubensdingen Abtrünnigen, sondern auch als potentiellen Mörder darzustellen, der in die Fußstapfen des ersten biblischen Mörders tritt. „Fasste man sein Verhältnis zu Jaakob gebildet auf [...], so war es die Wiederkehr und das Gegenwärtigwerden - die zeitlose Gegenwärtigkeit - des Verhältnisses von Kain zu Abel; und in diesem war Esau nun einmal Kain“ (S. 99). Dieser Kain trägt aber in der biblischen Geschichte weitaus problematischere Züge als Esau: Weil sein Opfer nicht so freundlich angenommen wird wie das seines Bruders Abel, ermordet er ihn. Esau schmiedet zwar Rachepläne, nachdem er das ganze Ausmaß der brüderlichen Infamie verstanden hat, er 
versöhnt sich aber, durch die Zeit besänftigt, ohne weitere Ansprüche mit Jakob.

Sooft wir Esau im Roman begegnen - und wir begegnen ihm einige Male in den ersten Kapiteln -, wird diese negative Einschätzung, die der Erzähler mit Jaakob teilt, verstärkt.

Ein ähnliches Vorgehen, mit umgekehrten Vorzeichen, wird für Jaakob angewendet. Schon bei der ersten Begegnung ist er ein würdiger Patriarch, er erscheint, begünstigt durch das Mondlicht, „von majestätischer und fast übermenschlicher Größe. [...] Noch eindrucksvoller [...] wurde seine Gestalt durch ihre Haltung [...]“" (S. 49). Er macht Eindruck auf alle, die ihn sehen: „Das war ein Herr und Gottesfürst, vornehm durch Geistesgaben, die auch seine gesellschaftliche Person veredelten" (S. 119). Was ihn aber besonders auszeichnet, ist sein Bewusstsein vom „Segen Gottes“, dessen Bedeutung er, im Gegensatz zu Esau, von Anfang an nicht hoch genug einschätzen kann. Wann immer er ängstlich, feige oder unterwürfig erscheint - z.B. in der Begegnung mit Eliphas, Esaus Sohn - (S. 97-103), so ist es, um „sein kostbares Verheißungsleben, für Gott und die Zukunft“" (S. 103) zu retten. Der Verlauf seiner späteren, genauso angstbesetzten Begegnung mit Esau, in der er, um seine „Unterordnung und Selbsterniedrigung“ (S. 108) zu demonstrieren, den Bruder schon im voraus mit Geschenken überhäuft, erklärt sich ebenfalls aus Jaakobs Bewusstsein um seine göttliche Auserwähltheit, in der er jeweils durch eine unmittelbar vorhergehende (Esau) oder nachfolgende (Eliphas) „Haupterhebung“ (S. 103) bestätigt wird. Jede weltliche Erniedrigung Jaakobs wird also begleitet durch eine „Erhöhung“, durch die seine Auserwähltheit, der göttliche Segen, bestätigt und bestärkt wird. Selbst seine unrühmliche Rolle beim Blutbad zu Sichem, bei dem er zunächst vorgibt, nicht zu wissen, was seine älteren Söhne planen, um sie dann nach getaner Tat zu verfluchen, weil sie sein „Haus samt Abraams Segen“ (S. 134) gefährdet hätten, vermag es nicht, das im Leser entstandene positive Bild von Jaakob ernstlich in Frage zu stellen.

Bei Jizchak wird eine andere Verschiebung vorgenommen. In der Bibel ist unbestritten, dass der Vater Isaak den göttlichen Segen auf Esau übertragen wollte, dass er aber, weil er blind war, die Intrige von Rebekka und Jakob nicht bemerkte. In dem Kapitel „Von Jizchaks Blindheit“ (S. 144) suggeriert uns der Erzähler, „daß Esaus kleiner und ertrotzter Lebensvorsprung von den Eltern niemals als ausschlaggebend verstanden worden“ (S. 146) war. Isaak „wollte es gern mit dem Erstling halten“ (S. 146), das war „,die Übereinkunft, die Isaak mit den Worten gegründet hatte und im Worte aufrechterhielt, ein kleiner Mythus innerhalb eines viel größeren und mächtigeren“" (S. 147) und - diesem widersprechend. Die Konstellation von zwei zur gleichen Zeit geborenen Söhnen hat es in der Geschichte seiner Familie noch nicht gegeben; den Segen bekam immer der Erstgeborene, der legitime Sohn der rechten Frau. Aber weder Charakter noch Lebenswandel Esaus sind geeignet, des Vaters ursprüngliche Bereitschaft, dem Älteren den Vorzug zu geben, die er „mechanisch, in spruchhaft feststehenden Wendungen“ (S. 146) äußert, zu 
verstärken. Schon im Vorfeld scheint also Jizchak unbewusst bereit, die Mechanik, den älteren nur wegen seines Erstgeburtsrechts zu segnen, zugunsten einer anderen Ahnenreihe zu unterbrechen, die über die Hirten Abel, Sem und Usir zu ihm selbst geht, und den Glatten und Häuslichen, nämlich Jaakob zu segnen.

Dass Jizchak deshalb erblindet war, ,weil er sich wohler in einem Dunkel fühlt, worin gewisse Dinge geschehen können, die zu geschehen haben" (S. 147), ist eine Behauptung, die der Erzähler ins Spiel bringt, selbst wenn er sie nicht abschließend zu werten scheint. Jizchak leidet zu sehr unter Esaus Lebenswandel, ,unter dem, was er mit ansehen oder wovor er die Augen verschließen musste, um es nicht zu sehen“ (S. 147). Der „kleine“ Mythus, wonach der Ältere Vaters Liebling und der Segensträger zu sein hatte, muss dem ,großen Mythus“ weichen, in dem der „wahrhafte“ Sohn gesegnet wird, und Isaak „lag im Dunkeln, auf dass er betrogen werde samt Esau, seinem Ältesten“" (S. 148).

\section{Die Rolle des Erzählers}

Der Erzähler ist im Josephsroman omnipräsent, dennoch ist seine Rolle durchaus nicht eindeutig. Er erzählt nicht nur, er bringt sich ein, wertet, kommentiert von einer zeitgenössischen Position her. „Im Unterschied zu dem traditionellen geschichtlichen und bibelgeschichtlichen Roman macht er sozusagen ,wissenschaftlichen Ernst' mit der Beschaffenheit seiner Quelle als eines auszulegenden, zu kommentierenden Textes [...].“5

Selbstverständlich ist der Erzähler völlig bibelfest; der Text ist gespickt mit Verweisen auf andere Bücher des Alten Testaments, auf die Psalmen, sogar auf neutestamentarische Stellen. Er zeigt sich als wissenschaftlich bewandert und durchaus auf dem Stand der zeitgenössischen kritischen Bibelforschung. Schon auf den ersten Seiten des Romans setzt er sich von einem naiven Verständnis der Urvätergeschichte ab: „Die Überlieferung will wissen“ schreibt er, um kurze Zeit später einzuschränken: „Das ist mit Vorsicht aufzunehmen oder jedenfalls recht zu verstehen. Es handelt sich um späte und zweckvolle Eintragungen, die der Absicht dienen, politische Machtverhältnisse, die sich auf kriegerischem Wege hergestellt, in frühesten Gottesabsichten rechtlich $\mathrm{zu}$ befestigen“" (S. 10). Er spricht von „Zusammenziehungen, Verwechslungen und Durchblickstäuschungen, welche die Überlieferung beherrschen" (S. 22).

Jene Überlieferung, die den gelehrten Kommentar eines Urtextes bildet, der seinerseits die späte schriftstellerische Fassung von Hirten-Wechselgesängen und schönen Gesprächen darstellt, weiß Übererfreuliches zu melden [...] Sie läßt sich um der Verherrlichung willen Übertreibungen zuschulden kommen, von

\footnotetext{
${ }^{5}$ Käte Hamburger, Der Humor bei Thomas Mann, Zum Joseph-Roman, München, Nymphenburger Verlagshandlung, 1969, S. 55.
} 
denen aber wir, zur endgültigen Klarstellung der Geschichte auch wieder nicht allzuviel abziehen dürfen, um nicht die Wahrheit neuerdings zu verrücken (S. 207).

Etwas herablassend übermittelt er andere biblische Geschichten, die er auch als „Hirtenmärlein“ (S. 128) bezeichnet: „Genau ist hier die wahre Reihenfolge der Geschehnisse zu beachten, die anders war, als später die Hirten im 'Schönen Gespräch' sie anordneten und weitergaben“" (S. 126). Aber nicht immer werden die Hirten korrigiert : „Ja, das alles stimmt nachprüfbar überein mit sich selbst und bezeugt auf die Dauer, dass kein Falsch ist in den Liedern der Hirten und ihrem schönen Gespräch“ (S. 270).

Überall (verrät) der Dichter die umfassenden Studie, die er, wie jeder historische Romancier, zur Realisierung der Begebenheit benötigte, andererseits aber auch das heimliche „Blinzeln“ [...] mit dem er den Ernst solcher Realisierung und eben damit auch die Überlieferung belächelt. ${ }^{6}$

Manchmal stellt der Erzähler etwas fest, um es gleich darauf wieder zu relativieren. So sagt er über Esaus Person: „Hiermit ist seine Persönlichkeit zweifellos fehlerfrei bestimmt, - zweifellos in gewissem Sinn, fehlerfrei unter Vorbehalt, denn die Genauigkeit dieser 'Bestimmung' ist die Genauigkeit des Mondlichts, der viel foppende Täuschung innewohnt [...]“ (S. 139).

Die Erörterung gehört hier zum Spiel, sie ist eigentlich nicht die Rede des Autors, sondern die des Werkes selbst, sie ist in seine Sprachspäre aufgenommen, ist indirekt, eine Stil- und Scherzrede, ein Beitrag zur ScheinGenauigkeit, der Persiflage sehr nahe und jedenfalls der Ironie: denn das Wissenschaftlichen, angewandt auf das ganz Unwissenschaftliche und Märchenhafte, ist pure Ironie.?

So erlaubt sich der Erzähler zu Esau die Bemerkung, dass ihn ,,die Lehrer und Wissenden viel heftiger beschimpften, als seine bürgerlich-irdische Person beschimpft zu werden verdiente“" (S. 143) - dabei ist es doch der Erzähler selbst, der in demselben Abschnitt Esau in die Nähe Ismaels, und diesen durch Austausch einer Silbe in die Nähe Samaels, d.h. des Teufels, rückt.

Dieses Erzählerverhalten hat natürlich Auswirkungen auf die Rezeption des Lesers. Der Erzähler ist nicht nur jemand, der es besser weiß als seine schriftlichen oder vorgeblichen mündlichen Vorlagen, er lässt auch dankenswerterweise den Leser an diesem Wissen teilhaben, er weiht ihn ein, erklärt und vermittelt. Seine Sprache ist „eine Sprache, wie ,du und ich' sie eben benutzen: Alltagssprache, nur eben etwas besser." 8

\footnotetext{
${ }^{6}$ Ibid., S. 65

${ }^{7}$ Thomas ManN, Gesammelte Werke in 12 Bänden, Bd. XI , Reden und Aufsätze 3, Frankfurt/Main, S. Fischer Verlag, 1960, S. 655.

${ }^{8}$ Bernd Jürgen FISCHER, Handbuch zu Thomas Manns „Josephsromanen“, Tübingen/ Basel, Francke, 2002, S. 125.
} 
Eine besondere Rolle spielt hier die unterschiedliche Verwendung des „wir“, bzw. „uns“. „Das ,Wir' des pluralis narratoris umfasst nicht nur jenen Erzähler, der manchmal fast alles und dann wieder kaum mehr weiß, als was auch schon in der Bibel steht." ${ }^{\text {99 }}$ In vielen Fällen handelt es sich um ein auktoriales Erzähler-Wir: „Wir geben uns keiner Täuschung hin über die Schwierigkeit, von Leuten zu erzählen, die nicht recht wissen, wer sie sind" (S. 94). Über die Geschichten Jaakobs kann er, im Bewusstsein seiner besseren Erkenntnis, schreiben: „Manches haben wir schon ausgebreitet und endgültig richtig gestellt" (S. 233). Aber nicht immer will der Erzähler ein abschließendes Urteil treffen: „Wir behaupten nicht, daß solche Ursache solche Wirkung zeitigen könne; wir begnügen uns damit, festzustellen, daß solche Ursachen vorhanden waren“ (S. 147). Manchmal muss der Erzähler zugeben, dass er auch nicht schlauer ist als seine Quellen: „Der Buchstabe der Überlieferung ist der einzige Anhalt, der sich uns bietet [...] Dennoch käme es uns nur zu, jene Deutung zu verwerfen und eine andere dafür einzusetzen, wenn wir eine bessere wüßten, was nicht der Fall ist" (S. 235).

An anderer Stelle impliziert dieses „wir“ den Leser, macht ihn zum Komplizen: „Darum sei unter uns gesagt und zugegeben“ (S. 89) - mit diesen Worten vertraut der Erzähler dem Leser sozusagen als Insiderwissen an, dass der Eliezer Jaakobs, wenn er von sich selbst als dem Diener Abraams spricht, nicht wirklich die gleiche Person meinen kann. Manchmal geht es um eine allgemeine Feststellung, die Erzähler und Leser teilen: „Sie genossen den schelmischen Übermut mit jener pädagogischen Verantwortungslosigkeit, die unser Verhältnis zu anderer Leute Kinder bestimmt“" (S. 283) heißt es zum Verhalten der Leute von Luz gegenüber Jaakob.

Den vielleicht schönsten Kommentar zu seinem oszillierenden Selbstverständnis liefert uns der Erzähler, als er von den Auswirkungen des Segens auf Jaakob und Laban schreibt:

\begin{abstract}
Was wir hier zu berichten haben, würde uns, wenn wir Geschichtenerfinder wären und es, in stillem Einvernehmen mit dem Publikum, als unser Geschäft betrachteten, Lügenmärlein für einen unterhaltsamen Augenblick wie Wirklichkeit aussehen zu lassen, sicherlich als Aufschneiderei und unmäßige Zumutung ausgelegt werden, und der Vorwurf bliebe uns nicht erspart, wir nähmen den Mund zu voll von Fabel und Jägerlatein, nur um noch einen Trumpf aufzusetzen und eine Lauschergutgläubigkeit zu verblüffen, die dann doch ihre Grenzen habe. Desto besser also, daß dies unsere Rolle nicht ist; daß wir uns vielmehr auf Tatsachen der Überlieferung stützen, deren Unerschütterlichkeit nicht darunter leidet, daß sie nicht allen bekannt ist, sondern daß einige davon einigen wie Neuigkeiten lauten. So sind wir in der Lage, unsere Aussagen mit einer Stimme abzugeben, die, gelassen, wenn auch eindringlich und ihrer Sache sicher, solche sonst zu befürchtenden Einwürfe von vornherein abschneidet (S. 208)
\end{abstract}

\footnotetext{
${ }^{9}$ Eckhard HEFTRICH, „Joseph und seine Brüder“, in Thomas-Mann-Handbuch, hrsg. von Helmut KoOPMANN, Stuttgart, Kröner, 2001, S. 466.
} 
Der Erzähler fordert Glaubwürdigkeit ein, gleichzeitig lässt er den Leser wissen, dass er das alles vielleicht doch nicht so ganz genau nehmen müsse. Er treibt ein schelmisches Spiel mit seinen Lesern, kokettiert mit seinem Wissen, das er auch dann als gesichert ausgibt, wenn dies nicht eindeutig ist, oder es dies' sogar eindeutig nicht ist.

\begin{abstract}
Die Genauigkeit, die Realisation sind Täuschung, ein Spiel, ein Kunstschein, eine mit allen Mitteln der Sprache, der Psychologie, der Darstellung und noch dazu der kommentierenden Untersuchung erzwungene Verwirklichung und Vergegenwärtigung, deren Seele, bei allem menschlichen Ernst, der Humor ist. ${ }^{10}$
\end{abstract}

\title{
Romanaufbau und Erzählstrategie
}

Damit die Umwertung der Segensintrige zum „großen Jokus“ gelingt, müssen aber zunächst alle die Elemente ausgebreitet werden, die zu dieser Umwertung beitragen. Der Leser muss bereit sein, dem Erzähler zu folgen, wenn dieser die Geschichte als vergnüglichen Vorgang hinstellt. Das wäre bei einem chronologischen Erzählen recht schwierig, steht doch diese Geschichte in der Bibel fast ganz am Anfang der Jakobsgeschichten.

Dort ist der Ablauf wie folgt: Die Zwillinge werden geboren (1. Mose 25, 20-28); Esau verkauft sein Erstgeburtsrecht (1. Mose 25, 29-34), es folgt ein Kapitel über Isaaks Leben, an dessen Ende zwei Verse über die HethiterFrauen Esaus stehen (1. Mose 26, 27), und dann wird auch schon der Segensbetrug erzählt, dem ein ganzes, vergleichsweise langes Kapitel gewidmet ist (1. Mose 27, 1-46).

Der Aufbau des Romans folgt diesem chronologischen Schema nicht, zumindest nicht in seinem ersten Teil. Das Kapitel „Der große Jokus“ steht an zentraler Stelle in den Geschichten Jaakobs, im vierten Kapitel des mittleren Hauptstücks. Es ist auch auffällig, dass es bis zu diesem Kapitel große Zeitsprünge vorwärts und rückwärts gibt, dass aber nach diesem Kapitel die Geschichten linear und chronologisch weitererzählt werden. Dass diese Erzählanordnung nicht zufällig ist und mit dem Sinn des Erzählten eng zusammenhängt, unterliegt keinem Zweifel. Sicher geht es zunächst darum, die „Grundzüge der Psychologie des mythischen Bewusstseins“ darzulegen, wie Hamburger schreibt. ${ }^{11}$ Aber der Erzähler benötigt auch Zeit, um die zentralen Personen so darzustellen, dass seine Interpretation des Segensbetrugs glaubhaft und logisch wird.

Welche Begebenheiten werden also vorrangig erzählt und wie erscheinen die Personen? Das erste Hauptstück beinhaltet ein „Schönes Gespräch“ zwischen Jaakob und Joseph. Jaakob ist hier schon ein ehrwürdiger Greis von 67 Jahren, der sein Leben Revue passieren lässt. Im Kapitel „Der Name“ (S.65) erinnert er sich an den langen Kampf am Jabbok und an den Namen

${ }^{10}$ MANN, Gesammmelte Werke, Bd. 11, S. 655.

${ }^{11}$ HAMBURGER, Der Humor bei Thomas Mann, S. 153. 
„Jisrael“, den er sich dabei errang. Im zweiten Hauptstück geht es zunächst um Abraam, der seine Frau Sara gegenüber dem ägyptischen Pharao als seine Schwester ausgibt, eine Geschichte, die im Buch Genesis in drei Varianten erzählt wird. Sie steht in unmittelbarer Nachbarschaft zum Kapitel „Mondgrammatik“. Der Erzähler beweist so, wie wir gesehen haben, dass die Identität der erwähnten Personen nicht fest umrissen ist, sondern sich in „Imitation und Nachfolge“ (S. 94) verschränkt. Im nächsten Kapitel sehen wir Jaakob gleich nach seinem Segensbetrug auf der Flucht; er wird von Eliphas, einem Sohn Esaus, gestellt und erniedrigt, so dass er nur sein nacktes Leben retten kann (S. 97). Diese Episode findet sich übrigens nicht im Buch Genesis; dort wird nur erwähnt, dass einer der Söhne Esaus Eliphas heißt. Bevor Jaakob zu seinem Onkel Laban weiterzieht, folgt eine Bestätigung des göttlichen Segens.

Dann folgt ein Erzählsprung von ungefähr 25 Jahren, vermittelt durch Jaakobs Erinnerungen. Zum ersten Mal nach dem Segensbetrug stehen sich Jaakob und Esau gegenüber. Esau ist noch immer der „gedanken- und bedeutungslose, zwischen Geheul und tierischem Leichtsinn schwankende Naturbursch von ehemals" (S. 108). Er macht seinem Zwillingsbruder ein großherziges Versöhnungsangebot, auf das Jaakob scheinbar eingeht, das ihm aber unannehmbar ist. Nach dem Zwischenspiel bei Sichem wird am Anfang des vierten Hauptstücks der Tod Isaaks geschildert. Am Totenlager des Vaters treffen Esau und Jaakob noch einmal aufeinander. Esau ist inzwischen völlig zu Edom, dem Roten geworden, und obwohl von seinem Versöhnungsverrsuch mit Jaakob schon die Rede war, wird hier noch einmal breit seine vorgebliche Annäherung an Ismael und das von diesem vorgeschlagene Doppelmordkomplott erzählt (S. 143). Ein Kapitel über „Jizchaks Blindheit“" (S. 144), dass das Verhältnis Jizchaks zu seinen Söhnen in einer für Jaakob sehr positiven Weise schildert, schließt mit den Worten: „Er lag im Dunkeln, auf daß er betrogen werde samt Esau, seinem Ältesten“ (S. 148).

Die Auslegung des Segensbetrugs als Riesenspaß ist nunmehr gut vorbereitet. Der Leser hat alles erfahren, was er zu Jaakobs Gunsten und insbesondere zu Esaus Ungunsten wissen muss. Jaakob ist durch den Segen reich geworden, reich nicht nur an Gütern und Söhnen, reich auch an Weisheit, Wissen und religiöser Überzeugung. Esau dagegen, zwar frühreif, doch entwicklungslos, ein grober Klotz ohne jegliches Bewusstsein einer göttlichen Sendung, steht in direkter Nachfolge zu den in der Bibel Verstoßenen, zu Kain und zu Ham. „Hier wurde der geistig Prädestinierte der Erbe, wenn es auch gewisser Umwege bedurft hatte, um den Segen von Esau, dem ,rechten Falschen', auf das Haupt des ,falschen Rechten', des Gesegneten selbst, zu lenken." ${ }^{\prime 12}$

Rebekka, die Mutter, eine dominierende Frau, die ihrem Liebling Jaakob öfter das Wort abschneidet, bereitet das verlangte Essen vor und verkleidet

${ }^{12}$ Ibid., S. 155. 
Jaakob, so dass er wie Esau wirken kann. Mit vor Angst klappernden Zähnen nähert sich Jaakob seinem Vater, und diese Angst ist gerechtfertigt, denn der Vater erkennt die Stimme nicht als Esaus Stimme. Jaakob versucht, so wenig wie möglich zu sprechen, aber er muss dem zweifelnden Vater Rede und Antwort stehen, bis dieser ihn „mit sehenden Händen“ (S. 154) als Esau anerkennt. Und als er schon gegessen hat, äußert er nochmals Zweifel: „Solltest du wirklich Esau sein, mein größerer Sohn?““ (S. 155) Mit letzter Kraft kann Jaakob antworten.

Zur Farce wird dieses Kapitel aber durch das Verhalten Esaus, der im Bewusstsein der auf ihn zukommenden Ehre alle Welt an seinem Stolz teilhaben lässt, bevor er das Wild für seinen Vater jagt. Bei seiner Rückkehr hat er natürlich, im Gegensatz zu allen anderen, nichts von dem Unheil mitbekommen. „In freudigem Dünkel und hochgebläht kam er daher [...]“ (S. 156). Alle sollen zusehen, wie er, der schon so gut wir Gesegnete, das Wildbret zubereitet. Und alle sehen ihm zu, nur dass ihr Lachen nicht Freude und Zustimmung ausdrückt, sondern Schadenfreude und Häme.

Der Moment der Anagnorisis wird für Esau zum schrecklichen, demütigenden Erwachen. Nicht nur, dass der Segen für immer von ihm fortgenommen wurde, dies geschah vor aller Augen, vor den Augen eines mitleidlosen, triumphierenden Publikums, in dessen scheinbarer Anerkennung sich Esau soeben noch gesonnt hatte.

Am Ende des Kapitels „Der große Jokus“ stehen die Leute um den um sein Erbe heulenden Esau herum und „hielten sich die Nieren“ vor Lachen, „so schmerzte sie der große Jokus, wie Esau, der Rote, geprellt ward um seines Vaters Segen“" (S. 158). Und der Leser lacht mit ihnen. Man gönnt diesem Esau seine Niederlage, als ob sie „eine angemessene Strafe für übles Tun wäre". ${ }^{13}$ Dabei ist dem Leser in diesem Moment sicher nicht bewusst, dass er an den geschickt geführten Fäden eines Erzählers genau mitten in den Kreis der lachenden Leute geleitet wurde, um mit ihnen den armen Teufel Esau auszulachen, der doch eigentlich nichts anderes getan hat, als sich ein wenig zu früh zu freuen.

\footnotetext{
${ }^{13}$ VON MATT, Die Intrige, S. 124.
} 